\title{
Enhancing Aesthetic Outcomes in Lower Eyelid Blepharoplasty
}

\author{
HESHAM A. HELAL, M.D.; NAHED S. BOUGHDADI, M.D. and HANY S. MAHMOUD, M.D. \\ The Department of Plastic Surgery, Faculty of Medicine, Ain Shams University
}

\begin{abstract}
Background: Blepharoplasty is one of the most commonly performed facial cosmetic procedures and can play a vital positive role in facial harmony and the perception of aging. Lysis of the orbicularis retaining ligament and of the orbicularis oculi insertion has been proposed to smooth the lid-cheek junction and tear trough. We aim to assess the usefulness and hazards of this procedure in comparison to the standard lower eye lid blepharoplasty.

Methods: 20 patients seeking lower eyelid rejuvenation; 10 having trans-cutaneous lower blepharoplasty with lysis of the orbicularis retaining ligament and of the orbicularis oculi insertion performed (group A) and the other 10 patients having standard trans-cutaneous blepharoplasty (group B). Final result, patients' satisfaction and complications were compared.

Results: Patients' age ranged between 30 and 65 years. Follow-up ranged from 2 to 16 months, with an average of 9 months. In group A: All patients reported excellent satisfaction with the aesthetic outcome, one patient developed early lower eye lid retraction which resolved spontaneously after 15 days with no other complications. In group B: 7 patients reported excellent satisfaction, while the other 3 showed mild improvement; two cases suffered lateral canthus rounding, one case had lid retraction which required lateral canthopexy.

Conclusion: The procedure seems to improve the harmony of the lid-cheek junction in the treatment of upper mid-face aging features
\end{abstract}

Key Words: Lower lid-Blepharoplasty.

\section{INTRODUCTION}

The peri-orbital area is always the main center of sight attraction as it is the most important points of the face that can express clearly many contradictors as youth beauty, aging, health or illness. Peri-orbital region usually shows the early signs of aging and is the most common complaint of the aged group of patients [1].

Concerning the lower eye lids; many procedures, either surgical or non-surgical, had been proposed for correction of the aging features that affect this region, however optimal results will not be achieved unless surgeons deal with not only eye-lids but also, and with the same importance, the usually present groove that separates the lower eye lid above and the mid-face below namely the lid-cheek junction [2].

The aging deep lid-cheek junction is usually presented clinically as a dark, deep hollow primarily caused by the mid-face descent together with laxity of lower eye-lid skin and fat prolapse due to the aging process [3]. The anatomical structures underlying that hollow have been investigated extensively in many studies and most of them agreed that it represents the attachment both the orbicularis retaining ligament laterally and centrally, and the tear trough ligament medially [4-7].

The orbicularis retaining ligament is a true collagen-elastin circumferential periorbital structure that originate in the lower eye lid from few millimeters below the infra-orbiral rim and inserts into the skin at the lid-cheek junction [8]. Being a part of the fascial apparatus of the face it acts as a fixing point to the orbicularis oculi muscle and lower eye lids in addition to its preventive role against spread of infection [9].

On the other hand, the tear trough ligament is also a true ligament that is present only medially starting from the medial canthus insertion and then extends laterally to merge with the orbicularis retaining ligament and is the main causative factor of tear trough deformity [10].

During lower eye lid blepharoplasty, the release of those two ligaments have been proposed in order to lift the mid-cheek and thus smoothen the lidcheek junction and tear trough zone [11]. We aim to assess the usefulness and hazards of this procedure in comparison to the standard lower eye lid blepharoplasty.

\section{PATIENTS AND METHODS}

This is a prospective, randomized comparative study that included 20 patients came to our clinic seeking rejuvenation of the lower eye lid. Full 
medical history is obtained and local clinical examination of the eye, and periorbital region to assess the aesthetic deformities present detect any medical problem that will harm the surgical operation.

The presence of lower eye lid deformity with no need for concomitant upper eye lid surgery was only inclusion criterion needed. Previous eye lid surgery, any ocular disorder and lid retraction are considered as the exclusion criteria of the study.

All the patients had lower eye lid blepharoplasty under general anaesthesia. They were divided into 2 sub-groups. 10 patients (Group A) had exposure through a sub-ciliary which is placed 3 to $4 \mathrm{~mm}$ below the lashes and beveled through the muscle to help preserve the pre-tarsal orbicularis function, then dissection of a skin-muscle flap is performed to the inferior bony orbital rim in order to expose the orbital septum and underlying fat pads. The septum is opened and only the excess fat from the 3 compartments was removed and then tightening of the septum is done. The skin is then re-draped in a predominantly superior direction with a slight lateral vector and excised conservatively.

The other 10 patients (Group B) had the same steps as the $1^{\text {st }}$ group with lysis of the orbicularis retaining ligament and the orbicularis oculi insertion performed as follows; after the skin muscle flap is dissected, the arcus marginalis is incised, and a sub-periosteal pocket is dissected from beneath the tear-trough area laterally to the malar eminence. Then the orbicularis muscle and the lateral canthus were suspended on the lateral orbital rim using a 3-0 non absorbable suture is done followed by closure.

Standard photographs are obtained; close up and full face frontal and lateral view. Follow-up ranged from 4 to 18 months, with an average of 5 months where all patients were assessed as regard the complications and also regarding their opinion about the improvement after 6 months from the operation.

\section{RESULTS}

All the 20 patients included in the study were females, the mean age in group A was 42 (range $=32-61$ ) while it was 39.3 in group B (range $=27$ 54). The overall complication rate was $40 \%(\mathrm{n}=$ 8 ) distributed among the 2 groups; $3(30 \%)$ in group A and 5 (50\%) in group B (Figs. 1-6).

The complications found in group A were: 2 cases of ecchymosis that resolved spontaneously within a week and 2 patients with lid retraction with only one of them needed revision after 2 months under local anesthesia. The complications found in group B were: 4 patients with lid retraction that resolved spontaneously within a month postoperative and one case with ecchymosis on one eye that resolved within 2 weeks. Table (1).

Table (1): Complications $n$ the studied group.

\begin{tabular}{llcc}
\hline & Complications & Group A & Group B \\
\hline 1 & Ectropion & - & - \\
2 & Edema and Ecchymosis & 2 & 1 \\
3 & Epidermal inclusion cyst & - & - \\
4 & Hematoma formation & - & - \\
5 & Infection & - & - \\
6 & Lid retraction & 2 & 4 \\
\hline
\end{tabular}
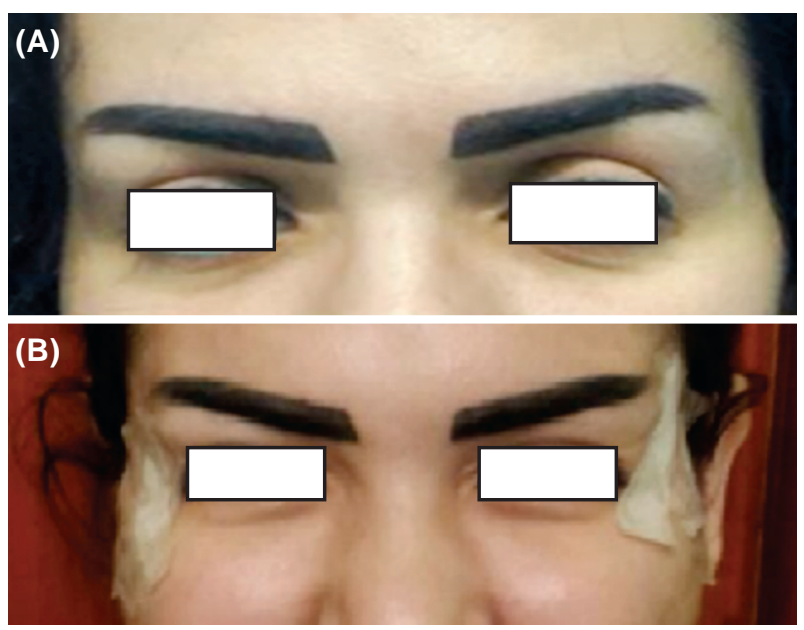

Fig. (1): Case number (1): A 21 year old female patient who underwent transcutaneous lower blepharoplasty frontal view pre-operative (A) and post-operative (B).

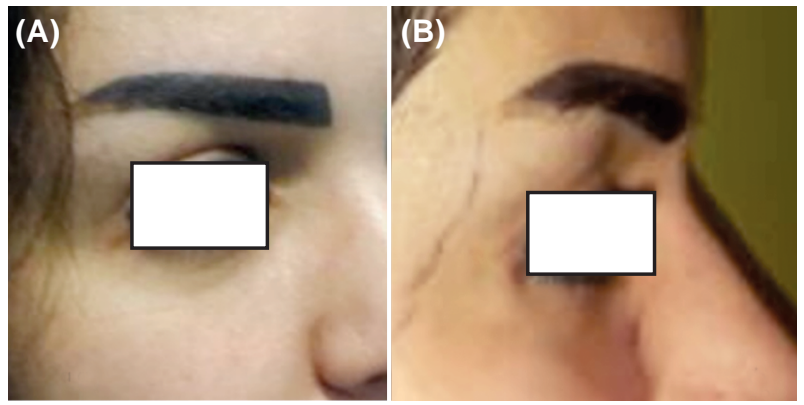

Fig. (2): Case number (1): Right lateral view pre-operative (A) and post-operative (B).

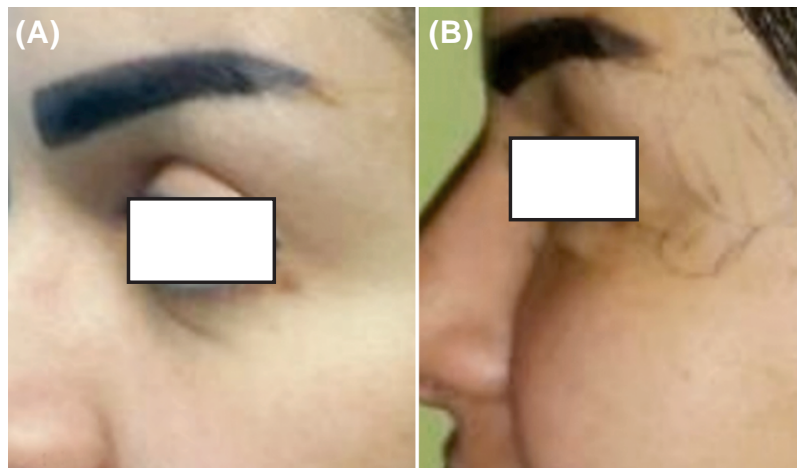

Fig. (3): Case number (1): Left lateral view pre-operative (A) and post-operative (B). 

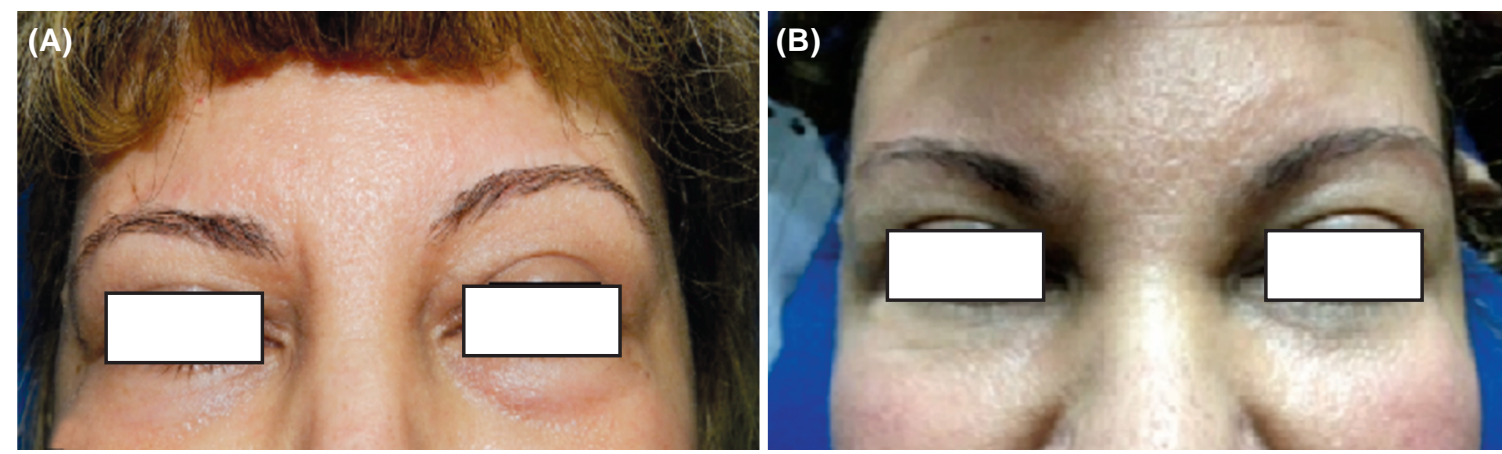

Fig. (4): Case number (2): A 42 year old female patient who underwent transcutaneous lower blepharoplasty frontal view pre-operative (A) and post-operative (B).
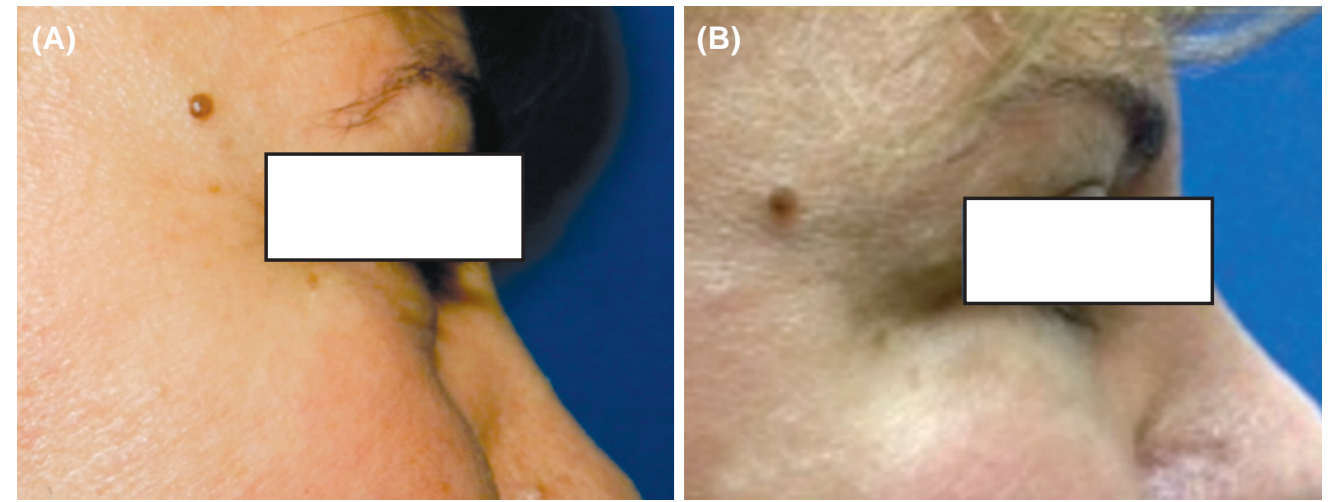

Fig. (5): Case number (2): Right lateral view pre-operative (A) and post-operative (B).
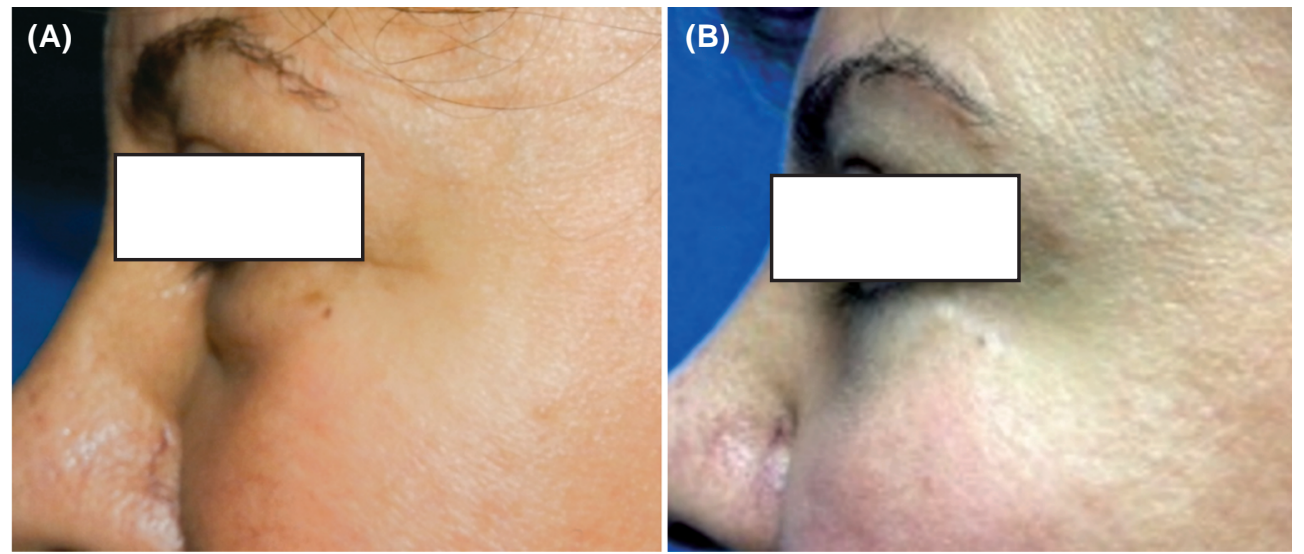

Fig. (6): Case number (2): Right lateral view pre-operative (A) and post-operative (B).

After 6 months after the operation all the patients reviewed their own pre- and post-operative photos and reported their opinion about the final improvement of their eye lid deformity. In group A; only 3 patients (30\%) reported their high satisfaction of the result compared to 7 patients $(70 \%)$ in group B and this showed to be of statistical significant differences between the 2 groups. The remaining 7 patients in group A showed moderate and mild satisfaction in 5 and 2 patients respectively. While in group B the remaining 3 reported moderate satisfaction and this was also of a significant difference between the 2 groups.

\section{DISCUSSION}

Blepharoplasty aims to correct signs of the aging face as tear drop deformity, pseudo-herniation of fat compartments, rhytides in addition to malar hollowing and thus creating a uniform contour and surface.

Rejuvenation of the lower eye lid without taking the transition zone between it and mid-face in consideration, will definitely affect the final appearance of the periorbital region as a whole. And thus if there is a technique that target the lid-cheek 
junction in conjunction with the surgical correction of lower eye deformity would be the technique of choice.

Many anatomical studies had proved the existence of the tear trough and the orbicularis retaining ligaments and their contribution in the aging features of peri-orbital region $[\mathbf{8 , 1 0 , 1 3}]$ and thus many authors had valuated the importance of their release during blepharoplasty to smoothen the lid-cheek junction $[\mathbf{7 , 1 1 ]}$. However, to our knowledge no study has been prospectively done to compare their superiority over other standard techniques.

Although many prefer trans-conjunctival approach over the transcutaneous one due to the increased risk complications, [14-16] we preferred the later in order to be able to conservatively correct any anterior lamella excess and properly tighten the lid. In all cases skin excision relatively small designed intra-operatively specifically to each one of them and with proper tissue handling, no excessive use of cautery, lateral canthopexy no one could fear of post-operative true ectropion.

Since first described by Goldberg, [17] fat repositioning has been widely used to soften deep tear trough areas during blepharoplasty, however in our study, we only minimally trimmed the excess fat in the 3 compartments and tightened the orbital septum to correct the eye lid bags as we think fat repositioning is technically time-consuming, not feasible for lateral compartment and add more suture material and also with proper release of the orbicularis oculi muscle insertion at the tear trough zone; there was no need for the transposition.

Enhancement of the mid face has been always a fixed patients' request in association with lower eye-lid rejuvenation especially with increased lidcheek distance. Adjuvant fillers has been used but the improvement is usually not lasting as other more sophisticated mid-face lift either through trans-oral or endoscopic or trans-temporal approaches.

With $70 \%$ of patients (7/10) in group B having high satisfaction rate compared to only 3 patients in group A, the newly described technique seems to offer a simple and efficacious method to enhance the aesthetic outcomes in lower eye lid blepharoplasty.

In our study, we reported higher rate of complication in group $B(n=5), 80 \%$ of them were lid retraction due to more extended dissection but with its rapid resolution with just manual massage we might classify it as prolonged normal recovery and not complication and this is supported by the nonaffection of the overall satisfaction rate up to 6 months after the operation.

Each eye lid has its own different group of aging deformities that will give it a specific picture and thus the technique for rejuvenation should be tailored accordingly. With the results of this comparative study, we think that this technique might be a good solution that would fit for most of the cases.

\section{Conclusion:}

This procedure seems to improve the harmony of the lid-cheek junction in the treatment of upper mid-face aging features.

\section{REFERENCES}

1- Grant J.R. and LaFerriere K.A.: Periocular rejuvenation: lower eyelid blepharoplasty with fat repositioning and the suborbicularis oculi fat. Facial Plast. Surg. Clin. N. Am., 18: 399-409, 2010.

2- Lambros V.: Observations on periorbital and midface aging. Plast. Reconstr. Surg., 120: 1367-1376; discussion 1377, 2007.

3- Carraway J.H.: Volume correction for nasojugal groove with blepharoplasty. Aesthetic Plast. Surg., 30: 101-109, 2010.

4- Kikkawa D.O., Lemke B.N. and Dortzbach R.K.: Relations of the superficial musculoaponeurotic system to the orbit and characterization of the orbitomalar ligament. Ophthal. Plast. Reconstr. Surg., 12: 77-88, 1996.

5- Pessa J.E., Zadoo V.P., Adrian E.K., Woodwards R. and Garza J.R.: Anatomy of a "black eye": A newly described fascial system of the lower eyelid. Clin. Anat., 11: 157$161,1998$.

6- Moss J.M., Mendelson B.C. and Taylor G.I.: Surgical anatomy of the ligamentous attachments in the temple and periorbital regions. Plast. Reconstr. Surg., 105: 14751490; discussion 1491-1498, 2000.

7- Haddock N.T., Saadeh P.B., Boutros S. and Thorne C.H.: The tear-trough and lid/cheek junction: Anatomy and implications for surgical correction. Plast. Reconstr. Surg., 123: 1332-1340; discussion 1341-1342, 2009.

8- Ghavami A., Pessa J.E., Janis J., Khosla R., Reece E.M. and Rohrich R.J.: The orbicularis retaining ligament of the medial orbit: Closing the circle. Plast. Reconstr. Surg., 121: 994, 2008.

9- Loeb R.: Fat pad sliding and fat grafting for leveling lid depressions. Clin. Plast. Surg., 8: 757, 1981.

10- Wong C.H., Hsieh M.K.H. and Mendelson: The tear trough ligament: Anatomical basis for the tear trough deformity. Plast. Reconstr. Surg., 129: 1392, 2012.

11- Schiller J.D.: Lysis of the Orbicularis Retaining Ligament and Orbicularis Oculi Insertion: A Powerful Modality for Lower Eyelid and Cheek Rejuvenation. Plast. Reconstr. Surg., 129: 692e, 2012. 
12- Ilankovan V.: Aesthetic blepharoplasty. British Journal of Oral and Maxillofacial Surgery, 48: 493-497, 2010.

13- Muzaffar A.R., Mendelson B.C. and Adams W.P.: Surgical anatomy of the ligamentous attachments of the lower lid and lateral canthus. Plast. Reconstr. Surg., 101: 873-884, 2002.

14- Baylis H.I., Long J.A. and Groth M.J.: Transconjunctival lower eyelid blepharoplasty: Technique and complications. Ophthalmology, 96: 1027-32, 1989.

15- Goldberg R.A., Lesner A.M., Shorr N., et al.: The transcon- junctival approach to the orbital floor and orbital fat: A prospective study. Ophthalmic. Plast. Reconstr. Surg., 6: 241-6, 1990.

16- Baylis H.I., Goldberg R.A. and Groth M.J.: Complications of lower blepharoplasty. In: Putterman AM, editor. Cosmetic oculoplastic surgery: Eyelid, forehead, and facial techniques. $3^{\text {rd }}$ edition. Philadelphia: WB Saunders, p. 429-56, 1999.

17- Goldberg R.A.: Transconjunctival orbital fat repositioning: Transposition of orbital fat pedicles into a subperiosteal pocket. Plast. Reconstr. Surg., 105: 743-8, 2000. 